\title{
Mais pourquoi continuent-ils à faire redoubler? Essai de compréhension du jugement des enseignants concernant le redoublement
}

\section{Géry Marcoux et Marcel Crahay}

Décider le redoublement d'un élève relève d'une décision complexe car il semble bien que ce soit un ensemble d'éléments qui conduit l'enseignant à considérer cette mesure comme celle qu'il convient de prendre. C'est cette complexité que cet article tente de saisir et d'analyser. Ce qui est proposé ici, c'est d'analyser plus finement l'action (processus et formation) qui conduit à ce jugement. Quels constituants fondent le jugement aboutissant à une décision de redoublement et quels poids prennent ces différents constituants dans la décision finale? Les données empiriques proposées sont issues d'entretiens menés auprès de 41 enseignants de Ge primaire dans le canton de Genève au cours de l'année scolaire 2007-2008. Partant pour un même enseignant de décisions effectives tantôt de redoublement tantôt de promotion, ces entretiens tentent de renouer la trame décisionnelle du jugement in situ. La question de la qualification de ce jugement comme "professionnel» est alors traitée dans la discussion finale.

\section{Introduction}

Envers et contre la succession de résultats de recherches concluant à l'inefficacité $\mathrm{du}$ redoublement, les enseignants de nombreux pays continuent à recourir à cette pratique. Faut-il n'y voir qu'incrédulité des praticiens face aux apports des Sciences de l'éducation? À la lecture d'enquêtes récentes (e.g., Draelants, 2006), cette explication parait réductrice. Celles-ci mettent en évidence la diversité et la complexité des facteurs qui rentrent en jeu dans la construction complexe de cette décision: ce serait l'interaction d'un ensemble d'éléments qui conduit l'enseignant à considérer cette mesure comme celle qu'il convient de prendre. Parmi ces éléments, les notes scolaires interviennent mais jamais de façon unique et pas toujours de façon prioritaire. Leur fonction consisterait, le plus souvent, à conforter un jugement en voie de formation, quand il n'est pas déjà formé. Ce qui semble alors déterminant, ce serait une série de perceptions d'inadaptation de l'élève eu égard à ce qui est attendu de lui en termes de participation en classe, d'attitude générale, de performances scolaires. Ces perceptions prendraient 
l'allure d'une évidence dans la mesure où les autres élèves, eux, répondent aux attentes. Bref, à l'origine du processus psychologique débouchant sur une décision de faire répéter l'année scolaire par un élève pointe une composante normative.

De quelle nature est le processus psychologique qui aboutit à des décisions de redoublement? Est-il possible d'élaborer ou même d'esquisser un modèle d'intelligibilité de ce processus intégrant les éléments qui y interviennent? Telles sont les questions au cœur du présent article.

D'autres recherches (Bless, Bonvin \& Schüpbach, 2005; Bressoux \& Pansu, 2003; Crahay, 2007; Hutmacher, 1993; Pini, 1991; Smith, 1990) menées sur le sujet ont déjà qualifié le processus à l'œuvre chez les enseignants de jugement, parfois de jugement professionnel. Leurs apports tendent à montrer que ce jugement porte davantage sur des dimensions psychologiques (e.g., maturité, attitude générale) que pédagogiques (e.g., maîtrise de certaines compétences). Y interviennent aussi des éléments connexes ou contextuels: l'histoire individuelle et/ou familiale de l'enfant, la situation actuelle de la famille, la disponibilité et le niveau de formation des parents et/ou des grands-parents, le contexte scolaire et notamment les caractéristiques du collègue qui pourrait accueillir l'élève en difficulté. De même, les enseignants auraient d'autant plus tendance à recourir au redoublement qu'ils adhèrent à une conception maturationniste du développement (Crahay, 2007; Smith, 1990; Tanner \& Combs, 1993; Tomchin \& Impara, 1992). Bref, le jugement conduisant les enseignants à se prononcer pour ou contre le redoublement d'un élève se révèle complexe par son modus operandi intrinsèquement multidimensionnel: avant de conclure, les enseignants prennent en compte une constellation d'éléments d'origine et de types divers. C'est donc cette complexité qu'il convient d'appréhender, d'analyser et, si possible, de modéliser leur jugement.

Ce qui est proposé ici, c'est d'analyser le plus finement possible le processus et la formation de jugements qui ont conduit des enseignants du primaire à décider le redoublement d'un élève. Dans ce dessein, l'analyse se ciblera d'abord sur les constituants de ces jugements aboutissant à une décision de redoublement. Tout en restant sur le plan qualitatif, il sera tenté d'appréhender le "poids» de ces différents éléments dans la décision finale. De nos observations antérieures (Crahay, 2007), nous savons que ces éléments sont soumis à une grande variabilité: le même argument peut être utilisé par un enseignant pour justifier le redoublement alors que, dans la bouche d'un autre, il sert à légitimer une décision contraire. Bien plus, on peut observer ce type de variabilité chez un même enseignant traitant de deux cas différents. Il s'agira donc d'essayer de comprendre pourquoi certains éléments (e.g., les conditions de vie familiale de l'enfant) poussent à la décision de redoublement dans certains cas et à la décision opposée dans d'autres. Enfin, il conviendra d'essayer de distinguer quels sont les éléments qui sont véritablement pris en compte dans le processus décisionnel et ceux qui servent à légitimer après coup la décision. 
Par sa focalisation sur le jugement des enseignants, le présent article s'inscrit dans une tradition scientifique qui, résolument enracinée dans la psychologie, repose sur l'idée de base selon laquelle «l'activité d'évaluation fait partie intégrante de nos modalités d'adaptation à l'environnement physique et social» (Aubret, 1995, p. 541). Autrement dit, il est proposé de reconnaître que l'évaluation scolaire mobilise des opérations mentales de traitement de l'information et, donc, des activités perceptives et cognitives. Selon Noizet et Caverni (1976), le noyau de ces opérations procède d'un schéma de comparaison, consistant à rapporter un objet à évaluer sur une échelle de valeur. Plus précisément, tout évaluateur sélectionne des observables qu'il va interpréter en fonction de systèmes de référence. Ainsi, évaluer le savoir-lire, c’est sélectionner certains indices ou indicateurs parmi une très grande diversité d'observables possibles (vitesse de lecture, fluidité de la lecture à haute voix, erreurs et hésitations, façons de reformuler les idées du texte, etc.) et les interpréter en termes de niveau. Le système de référence de l'individu intervient d'emblée dès l'étape de sélection des observables et influe également sur l'interprétation de ceux-ci. Dans cette perspective, il est légitime de s'interroger, comme le fait Gilly (1980), sur les mécanismes sous-jacents aux jugements portés par les enseignants sur les élèves. Selon cet auteur, l'enseignant perçoit l'élève à travers un cadre schématique qui est à la fois sélectif, organisateur et interprétant des informations disponibles. Or, ce cadre est façonné par les objectifs professionnels et les normes socio-institutionnelles qui habitent chaque praticien. Plus précisément, les jugements des enseignants sont le produit des rapports entre trois déterminants: on trouve, à un premier niveau, l'expérience quotidienne (activités, indices comportementaux, etc.), au niveau intermédiaire, les représentations sociales (l'écolier type, le bon élève, etc.) et, à l'autre extrémité, les normes sociales générales (valeur morale, modèle de l'homme dans la société, etc.) et les normes scolaires institutionnelles (règles de fonctionnement de l'école, objectifs, etc.). Par conséquent, la perception de l'élève par l'enseignant est socialement et institutionnellement déterminée.

\section{Méthodologie}

Les données empiriques examinées ci-dessous sont issues d'entretiens menés dans le canton de Genève. Partant de décisions effectives de redoublement prises par des enseignants primaires, ces entretiens tentent de retracer la trame décisionnelle du jugement in situ. Une attention particulière est portée aux éléments a priori secondaires: critères déontologiques et éthiques, croyances en matière d'enseignement et d'évaluation (Laveault, 2005). Notons d'emblée que le matériau d'analyse provenant d'enseignants du primaire, il conviendra d'éviter toute généralisation aux enseignants du secondaire ou d'autres niveaux scolaires: rien ne permet d'affirmer que les processus psychologiques sont identiques. 
Au total, ce sont les entretiens de 41 enseignants genevois de 6e primaire (30 femmes; 11 hommes) qui ont été analysés. Ces entretiens ont été réalisés entre octobre et décembre 2007. Le nombre d'années d'enseignement varie de 2 à 35 $(M=13.2 ; E T=10)$.

Chaque enseignant a été interrogé selon le même protocole. Après quelques questions d'identification, il lui était demandé dans un premier temps de se remémorer le cas d'un élève qu'il avait fait redoubler et de retracer si possible la construction de sa décision. Dans une deuxième partie d'interview, il était cette fois sollicité sur le cas d'un élève qui aurait pu redoubler, mais pour lequel il avait pris une décision de promotion. Dans chaque cas, le chercheur s'intéressait à la place des évaluations dans cette décision ainsi qu'à celle d'autres facteurs, au sentiment de l'enseignant sur le bien-fondé de sa décision a posteriori et à ses croyances sur le redoublement.

Ces enquêtes privilégient dès lors l'émergence des conceptions et croyances ${ }^{1}$, des principaux acteurs de cette décision: les enseignants. Nous avons choisi de réaliser principalement une analyse thématique, formelle (formes et enchaînement du discours) et structurale (aspects sous-jacents et explicites du message). Les résultats sont ainsi à prendre comme résultant d'études de cas et leur analyse comme clairement qualitative au sens de Crossley et Vulliamy (1997).

\section{Résultats}

L'analyse thématique de la totalité des entretiens telle que nous l'avons menée permet de faire émerger quatre blocs de commentaires. Ceux-ci seront analysés successivement.

- Bloc 1: L'importance des résultats scolaires (atteinte des objectifs)

- Bloc 2: L'influence d'un construit représentationnel que nous proposons de nommer "Profil élève et ses ressources»

- Bloc 3: L'influence des conceptions pédagogiques (conceptions de l'évaluation et croyances aux bienfaits du redoublement)

- Bloc 4: Les problèmes moraux et éthiques avec prise en compte des contraintes et recherche de soutiens extérieurs

\section{De l'importance des résultats scolaires (atteinte des objectifs)}

Si un peu plus de la moitié des enseignants interrogés [23/41 - 56,1\%] déclarent explicitement que les évaluations ont pris une place importante dans leur décision de promouvoir ou de retenir l'élève en difficulté, un bon tiers [15/41 $36,6 \%$ ] déclare qu'elles n'y ont pris que peu de place. Six enseignants se contredisent même clairement sur ce point entre les deux parties de l'entretien. Quoi qu'il en soit, leur rapport à la note scolaire est stratégique. La citation de deux propos d'enseignants est éclairante à ce sujet. 
Je lui ai mis 3 pour qu'elle parte; soyons clairs! [Entretien 1]

En étant très gentil, on pouvait éventuellement pousser le 2 au 3. On ne regardait pas l'orthographe, on ne regardait pas ci... Donc c'est clair qu'on peut toujours tricher. Je veux dire, les notes c'est les notes; il n'y a personne qui va venir vérifier ce qu'on va mettre dans notre registre. Mais en même temps ce que je dis toujours aux élèves et aux parents: A quoi ça sert de tricher, pour retomber et se faire plus mal? C'est-à-dire qu'à un moment donné, il vaut mieux se dire: Ok, là ça vaut un vrai 2; on sait où on en est. Plutôt que de se dire: On va mettre un 3 par complaisance; en disant: On pousse, on pousse, on pousse. Et puis d'arriver, parce que 3 c'est presque atteint. Donc c'est vrai que les résultats scolaires, c'était ce quills étaient et puis voilà. [Entretien 58]

Bien sûr, la prise en compte de la progression de l'élève, même si elle n'est citée explicitement que par seulement treize enseignants, se retrouve systématiquement à l'analyse de chaque discours singulier. Par exemple, un enseignant déclare:

Dans les évaluations formelles on voyait clairement que les bases nétaient pas maîtrisées. À partir de ce moment-là, on est confronté à un dilemme: on se dit que de toute façon, objectivement parlant, l'enfant n'a pas atteint ses objectifs; mais en même temps, par rapport à là d'où il vient, il a progressé. [Entretien 52]

Ce verbatim a l'avantage d'exprimer également un dilemme entre progression réelle et non atteinte des objectifs. Il semble, en définitive, qu'un élève peut avoir été en échec tout au long de l'année, mais doit - pour être promu - au moins présenter un progrès pendant le dernier trimestre; cette évolution positive peut lui être créditée même si le résultat final se situe juste en dessous du niveau attendu. Cette prise en compte de l'évolution de certains élèves, tout à fait explicite chez certains enseignants, traduit un pari sur l'avenir qui peut se doubler d'une peur de se tromper et de la crainte de briser un élan naissant. Il faut d'emblée préciser que ce pari positif dépend de ce que nous proposons d'appeler ciaprès le «profil de l'élève et de ses ressources familiales».

\section{... en interaction avec un construit "profil d'élève et ressources"}

Il serait erroné de déduire de ces premiers résultats que les enseignants décident du redoublement des élèves sur la seule base des notes scolaires, de l'atteinte des objectifs et/ou de leur progression scolaire en cours d'année. Plus que ces éléments relevant de la sphère des apprentissages, la représentation que l'enseignant s'est construit de l'élève intervient. Cette représentation consiste en un profil de l'élève construit en référence à l'adéquation de son comportement en classe au «métier d'élève» (Perrenoud, 1994) tel que l'enseignant le conçoit. De nombreux propos d'enseignants pourraient être cités à l'appui de cette analyse. On se contentera de quelques-uns. 
Tu dois toujours faire un profil global de l'élève et prendre tout en compte. Tu ne prends pas seulement les notes finales. [Entretien 38]

Un élève qui suit en classe, se respecte, respecte les cours et les maîtres qui les donnent, fait ses devoirs correctement est rarement inquiété. La relation de confiance à l'école et aux maîtres est à mon sens déterminante pour la réussite. [Entretien 21]

[...] une grande part d'autonomie, à mon avis, c'est un facteur très important surtout pour le cycle après: un des plus important, je pense. Il faut aussi savoir sorganiser, prendre en charge son travail suffisamment. Je pense aussi qu'il est important de ne pas toujours être en difficulté, mais de pouvoir aussi réussir seul, se débrouiller pour faire une tâche, sans aide. [Entretien 48]

D’une manière générale, le métier d'élève tel qu'il doit être assumé implique, aux yeux des enseignants, de l'autonomie dans la prise en charge des tâches scolaires, une attitude générale témoignant d'une motivation positive et d'un engagement réel par rapport aux apprentissages. Le rythme de travail - c'est-à-dire la lenteur pour les redoublants - est également pris en compte.

Les capacités cognitives (principalement sa capacité de mémorisation et sa capacité de raisonnement) interviennent également dans la construction du profil.

Il y avait ça... un manque de maturité par rapport au raisonnement mathématique [...]. Il y avait la conjugaison: ça ne rentrait pas; la grammaire où il y a aussi une forme de logique; l'orthographe... C'était un peu tout. [Entretien 14] [...] elle se donnait une peine folle, pour apprendre par cour, donc elle faisait tout juste. Mais dès qu'il y avait du raisonnement, elle n'y arrivait pas. [Entretien 30]

Il est remarquable de constater que des enseignants lient capacités cognitives et caractéristiques biologiques de l'élève et, plus précisément, sa maturité, son âge, sa taille et/ou sa corpulence. Il est évidemment tentant de lire dans certains propos d'enseignants et d'autres l'expression d'une conception maturationniste du développement.

Il était grand en taille; l'autre élève dont nous parlions avant était petit. Physiquement je pense qu'il y a des enfants qui peuvent bien vivre un redoublement comme il y en a d'autres qui sont trop... [Entretien 55]

D'office, il [l'inspecteur] mavait dit que vu son jeune âge et vu sa maturité, elle allait doubler. [Entretien 18]

Dès lors, toutes choses égales par ailleurs, être plus jeune que l'âge moyen de l'année scolaire ou en avance semble pénaliser l'élève au moment où l'enseignant doit prendre la décision de promotion alors qu'être en retard (c'est-à-dire avoir déjà vécu un redoublement) devient une circonstance favorable à la réussite, et ceci sans prise en considération de l'effet espéré du redoublement. 
C'est assez contradictoire, mais je ne la voyais absolument pas refaire une Ge, malgré ses [notes] 2. Parce qu'elle est allée au bout de ce qu'elle pouvait faire, dans son cas, dans les conditions qu'elle était. Mais là, elle n'aurait pas été dans des conditions bonnes pour refaire, elle est trop grande, elle est trop en décalage. Ça, ça compte vachement aussi! [Entretien 1]

De même, l'élève est rarement considéré seul. Bon nombre d'enseignants prennent en compte le profil de la famille, ce qui inclut le niveau socio-économique perçu, les histoires familiales actuelles ou passées, les niveaux intellectuels individuels des deux parents, leur fragilité ou force psychologique, leur implication individuelle dans l'école et le soutien scolaire apporté ou potentiel ainsi que leur refus-acceptation de la possibilité d'un redoublement. L'enseignant porte alors de facto un jugement sur la famille; celui-ci s'accompagne d'une évaluation quant à la capacité de celle-ci à offrir à l'enfant l'appui estimé nécessaire à un passage de classe malgré les faiblesses constatées.

Moi, je dirais que dans les deux cas, pour celle qui a réussi, elle avait quand même une famille, des parents qui étaient là pour l'aider derrière et la soutenir, qui avaient tout mis en place pour que ça fonctionne. De l'autre côté, les parents, j'avais l'impression... quills ne se rendaient pas compte de l'impact que ça avait sur l'enfant du fait qu'il redouble. Ils n'ont pas mis grand chose en place. Pourtant, ils pouvaient avoir les moyens, ce n'était pas une faute de moyens. [Entretien 8] Et il y a la question du soutien. Plus l'élève est soutenu mieux c'est. Mais ça, ça dépend aussi du milieu socio-économique; tous les enfants ne bénéficient pas $d u$ même soutien. [Entretien 24]

Il y a trois choses: un niveau social qui aide; des parents qui peuvent aider et jeter un coup d'ail à ce quil fait, qui comprennent notre charabia, qui sont à la maison. Deux, il y a les capacités intellectuelles mais je crois que tous les ont, mais $X$ a mieux réussi à les mettre en application $q u$ 'Y du fait des parents, de la répétitrice. Et puis en trois, le travail et la motivation du travail. Ces trois points se complètent et dans le cas de $Y$, la mère n'a pas suivi. Je pense que si les parents ne sont pas là pour recadrer l'enfant, à cet âge, c'est dur pour l'enfant de se responsabiliser tout seul. [Entretien 50]

Intervient enfin, dans cette évaluation du potentiel familial, leur ouverture à l'idée de recourir au soutien de spécialistes (logopède, pédopsychiatre, ...). Car, en définitive, lorsque les enseignants évoquent le profil d'un élève, ils opèrent un assemblage de perceptions et de présupposés qui renvoie à la fois aux caractéristiques propres de l'enfant et au potentiel éducatif de son entourage. Ayant à décider de la promotion ou du redoublement des élèves, les enseignants troquent leur habit d'activateur des apprentissages pour celui de psychologue (Crahay, 2007), convoquant à la barre de la délibération des arguments relevant tantôt d'une conception maturationniste du développement, tantôt d'une psychologie systémique ou écologique. Il semblerait que les enseignants peuvent activer soit 
un raisonnement de compensation-correction, soit un raisonnement cumulatif. Il arrive, en effet, que des enseignants justifient la promotion d'un élève considérant que le potentiel de son écosystème (ses parents, grands-parents ou autres personnes de son entourage) est susceptible de compenser ses faiblesses personnelles. À l'inverse, pour légitimer une décision de redoublement, des enseignants font souligner que les caractéristiques «négatives» du milieu viennent se surajouter aux manquements individuels. Ce serait principalement dans ces cas de figure que les enseignants recourraient à des arguments maturationnistes, invoquant l'âge, la taille ou la corpulence de l'enfant.

\section{L'influence des conceptions pédagogiques}

De multiples propos d'enseignants traduisent la persistance de croyances sur les effets bénéfiques du redoublement. Celui-ci reste majoritairement vu comme une seconde chance. De manière synthétique, recommencer présenterait différents avantages liés au fait général de donner un supplément de temps. Ainsi, il serait bénéfique de donner du temps aux enfants pas assez mûrs, car on suppose que, durant l'année de redoublement, la maturité va s'acquérir. Il serait également bénéfique de donner du temps aux enfants qui ont des situations familiales difficiles à gérer: on protège ces enfants en ne rajoutant pas une difficulté supplémentaire à leurs problèmes. Le redoublement serait également une manière d'éviter une perte de confiance en soi (par rapport à une promotion qui l'affecterait nécessairement) ou d'aider à la restaurer par la répétition d'activités déjà connues, ce qui réduit la charge cognitive ou la «charge de travail» de l'élève et devrait contribuer à le rassurer sur ces capacités.

Le redoublement, je le vois plutôt comme une deuxième chance. [Entretien 50] Comme il avait déjà vu le programme une fois, il était plus à l'aise parce qu'il avait plus confiance en lui: les parents l'ont laissé un petit peu respirer. [Entretien 14]

[...] car souvent les parents ne veulent pas réduire la charge de travail. Ils ne veulent pas que leur enfant ait du retard. C'est une illusion! Les parents raisonnent à l'envers car le passage entraîne quelques minutes de bonheur et de nombreuses années de labeur. Mais je laisse les parents décider en les avertissant de ce qu'ils vont vivre avec leur préadolescent. [Entretien 11]

S'appuyant sur une conception pédagogique selon laquelle l'apprentissage se fait de façon linéaire avec emboîtement des connaissances brique après brique, des enseignants expliquent que le redoublement permet de récupérer les lacunes et de consolider les bases non encore acquises.

[...] l'élève obtient vraiment que des 2 et là on lui laisse une chance pour qu'il puisse rattraper ses lacunes. [Entretien 1]

Reposant également sur cette conception cumulative des apprentissages scolaires, bon nombre d'enseignants croient aux bienfaits du redoublement précoce. 
Je pense qu'il vaut mieux faire refaire les petites classes pour consolider les bases... C'est une chance qu'on leur offre pour pouvoir se reprendre et se sentir autrement que toujours à la traîne. [Entretien 11]

Lisant la réalité ou l'interprétant en fonction de cette croyance dans les bienfaits du redoublement, les enseignants tirent parti de diverses observations pour renforcer leur conviction. C'est le cas, d'une part, quand un élève faible promu ne va pas mieux et lorsque le redoublement se passe bien.

Elle est de nouveau en échec cette année et elle se sent dévalorisée, mais elle ne fait pas l'effort de changer d'attitude, voire de faire ses devoirs de manière satisfaisante. [Entretien 21]

En tous cas, là, pour le moment, ça à l'air d'être plutôt positif pour lui, ça contredit d'ailleurs beaucoup ce que je pensais. [Entretien 48]

Les contre-exemples ne les perturbent pas nécessairement: quand l'élève promu va bien, des enseignants se demandent si son évolution n'aurait pas encore été plus marquée avec redoublement.

Oui et non. Oui, car il s'en est sorti et je le vois, il a l'air bien. Puis mon collègue qui l'a aujourd'hui me dit qu'il ne s'en sort pas trop mal malgré qu'il soit un peu lent et qu'il ait quelques difficultés. Mais après tout, je n'en sais rien. Je ne sais pas ce qui se serait passé s'il avait redoublé. Il aurait peut-être pu consolider ses acquis et reprendre le programme à son rythme. Ça aurait peut-être été plus bénéfique. [Entretien 24]

Quand le redoublement ne fonctionne pas, il est toujours possible de mobiliser une attribution externe à l'enseignant: l'élève n'a pas consenti les efforts nécessaires ou il y a manque de soutien parental, etc.

D’une manière générale, si la croyance des enseignants dans les bienfaits du redoublement est une réalité qu'il est impossible de nier, celle-ci est conditionnelle. Le redoublement est efficace si... Deux propos d'enseignants permettent d'illustrer ce constat majeur:

[...] l'enfant doit être motivé, les parents doivent être motivés et il faut qu'il y ait un soutien derrière. [Entretien 55]

Je suis pour du moment que les parents et l'élève ont pris conscience de ce changement. [Entretien 8]

Il s'agit donc bien là de croyances, car l'argumentation opposée est logiquement tout aussi défendable. Les enseignants pourraient tout aussi bien concevoir que la promotion est favorable si parents et enfants saisissent cette opportunité comme une chance qui leur est offerte de rebondir. 


\section{Des Dilemmes éthiques ... en prise avec des contraintes institutionnelles divergentes}

La croyance des enseignants dans les bienfaits du redoublement est donc bien réelle, même s'ils spécifient des conditions liées essentiellement à la réactivité de l'élève et de son entourage social. Leurs doutes semblent majoritairement liés à une autre question: qui est-il juste de faire redoubler? Ce questionnement débouche régulièrement sur des dilemmes moraux, derrière lesquels on voit poindre deux valeurs cardinales: le mérite et le talent.

Les propos d'un enseignant, généralement enclin à éviter le redoublement, est particulièrement éloquent à ce sujet.

Pour le moment, c'est le seul élève pour qui j'avais l'impression que c'était une bonne chose qu'il redouble. Donc de toutes les années, j'ai fait doubler un seul élève. En fait je ne peux pas dire si je suis pour ou contre, mais c'est la seule fois où je me suis dit que c'était nécessaire. Il y a aussi eu un autre élément qui a influencé mon idée, c'est que l'année d'avant j'avais eu une autre élève, qui avait aussi beaucoup de difficultés, mais qui, elle, se donnait une peine folle, pour apprendre par cour. Donc, elle faisait tout juste mais dès qu'il y avait du raisonnement elle n'y arrivait pas. Et elle, on l'avait fait doubler dans l'école. J'avais vraiment le sentiment d'injustice par rapport à cette élève qu'on a fait doubler mais qui travaillait tellement. Mais elle ne pouvait pas réussir car elle était limitée. Puis par rapport à lui qui était un "glandu» et qui ne faisait rien. Je pense que ça a un peu aussi influencé ma décision. [Entretien 30]

Pour rendre compte de la diversité des points de vue, il importe d'être nuancé. Il semble bien que tous les enseignants soient tenaillés par un questionnement concernant ce qui est juste de faire, mais ils y répondent différemment. Certains enseignants ne s'autorisent pas à évaluer différemment les élèves en difficulté au nom de l'équité, alors que d'autres se le permettent au nom du même principe. Quelques extraits d'entretien illustrent la variabilité des positionnements.

Ben oui, parce que c'est à partir de là qu'on se base. C'est clair que l'on se dit: Il a des difficultés, on devrait peut-être l'évaluer différemment que les autres. Mais en même temps on a certains objectifs à suivre et puis on ne peut pas... Enfin, je veux dire: Après, il y a aussi les épreuves communes auxquelles il a eu des résultats catastrophiques où on se rend bien compte que les acquis ne sont pas là. [Entretien 15]

C'était vraiment un élève qui se donnait de la peine. Il faisait beaucoup d'efforts pour remonter la pente. Et, je voyais ses progrès. [Entretien 24]

On ne s'est pas vraiment basé sur les évaluations parce qu'on voyait bien que la faire doubler n'aurait servi à rien. [...] Comme elle avait déjà deux ans de plus, nous ne pouvions pas la garder. [Entretien 17]

La décision est probablement simple avec les cas extrêmes: d'un côté, ceux qui ont le talent ou les capacités et le mérite et, de l'autre, ceux qui n'ont aucun des 
deux (l'incapable paresseux). Mais que faire face à ceux qui sont méritants, mais peu doués et aux autres qui, moyennement doués, sont des "glandus»?

Et lorsque le doute s'insinue dans le jugement de l'enseignant, que fait-il? Comme tout autre être humain sans doute, il cherche du soutien. Auprès de collègues, de spécialistes (logopédiste ou psychologue), de l'autorité (l'inspecteur ou le DIP), voire même des parents. Certains en appellent alors à des critères de décision «objectifs» et/ou à des procédures rigoureuses c'est-à-dire à une évaluation la plus fiable possible qui permettrait de trancher objectivement pour les conforter et les rassurer dans leur choix. C'est manifestement le cas de l'enseignante suivante:

Je me suis retrouvée dans une situation où je devais décider d'un redoublement sans avoir à aucun moment une justification de la part de l'évaluation. J'étais juste à mettre un 2 à l'épreuve cantonale ou un 3. Et le pire, c'est que quand j'ai demandé au secteur de l'évaluation où ils mettaient la limite entre le 2 et le 3, ils n'ont pas voulu me répondre. [...] Puis, ils mont dit: Oui mais c'est vous qui devez voir comment elle a évolué au cours de l'année. Je leur ai dit: Oui mais il y a quand même des objectifs à atteindre [...] Si on fait des épreuves cantonales c'est pour juger tous les enfants de la même façon; donc à un moment il faut nous dire où est la limite. Je m'en fiche de savoir où est la limite entre le 5 et le 6 ou le 4 et le 5, mais par contre quand il s'agit de faire doubler un enfant ou pas... [Entretien 1]

Pour ces enseignants genevois de 6P dont les évaluations finales vont déterminer l'orientation des élèves dans l'une ou l'autre filière, certains dilemmes prennent un accent tout particulier en raison d'une différence fondamentale qui existe entre enseignement primaire et cycle d'orientation (ou collège si on se réfere à la situation française). L'enseignant primaire, porté par l'habitus propre à sa corporation, tend à privilégier la progression des élèves alors qu'au cycle, la tendance consiste à ne prendre en compte que le résultat final.

Pour le cycle de toute façon c'est les résultats finaux qui sont intéressants. Enfin, ils regardent ça eux, ils regardent le carnet. Et puis en ce qui nous concerne, l'inspectrice ou avec les autres collègues, ce qui est important c'est de voir ce qui se passe aussi en classe: l'investissement, les progrès qu'ils font peut-être dans les exercices, qu'ils ne font peut-être pas forcément en situation d'examen. Mais... en Ge primaire de toute façon les évaluations ça a un poids énorme [...] Donc, oui les choses qui se sont passées en classe ont eu de l'importance, mais au final, quand ils vont se présenter au cycle, c'est la dernière page du carnet qu'ils montrent avec les résultats obtenus puis éventuellement les commentaires. Mais voilà, je ne pense pas que ça soit ça qui fasse vraiment du poids dans leur décision. [Entretien 62]

Cette connaissance du fonctionnement différent du cycle incite certains à des raisonnements stratégiques, mais toujours au nom de l'intérêt de l'élève.

Mais au cycle, je leur ai dit que de toute façon, elle n'y arrivera pas plus l'année prochaine. J'ai dit: Elle, je pense qu'il faudrait la mettre en C. Mais de toute fa- 
çon, on ne l'a prend pas. Alors j'ai dit: Mais qu'est-ce qu'on va faire? Moi, je pense que ça va être une année de perdue parce que l'année prochaine, elle ne va rien apprendre de plus. Mais non...vous savez, ça ira mieux. Elle va refaire. Je pense ça va.... Ben eux, je pense qu'ils ne les prennent pas et puis voilà: on ne négocie pas. C'est comme ça. Ce sont les règles. Et moi je ne les connaissais pas ces règles. Maintenant je les connais. Et je pense que cette élève, je lui aurais mis des notes pour qu'elle passe en $C$, parce que là, elle va perdre une année. [Entretien 13]

\section{Discussion et conclusion}

Dès lors qu'il s'agit de se prononcer pour ou contre la promotion d'élèves en difficulté, les enseignants mobilisent des jugements évaluatifs, au sein desquels opèrent en leur centre une croyance conditionnelle dans les bienfaits potentiels du redoublement. Cette croyance s'appuie, sur le plan rationnel sur une conception psychopédagogique concernant la séquentialité des apprentissages et leurs nécessaires enchaînements. Convaincus qu'un apprenant qui ne maîtrise pas les bases ne peut plus rien bâtir de solide en matière de connaissances et de compétences, les enseignants pensent agir dans l'intérêt des élèves en leur imposant de répéter une année dès lors que leurs lacunes sont criantes. Autrement dit, sans être efficace à tous les coups, le redoublement serait la «meilleure» solution ou la moins mauvaise (un "pis-aller») ou encore la seule envisageable lorsqu'un enfant paraît "perdre pied», dépassé dans la plupart des apprentissages par le rythme de la classe, alors que la majorité des autres réussissent à suivre ce rythme. La croyance dans les bienfaits du redoublement peut également s'accommoder d'une conception maturationniste du développement: l'enseignant arguera alors que la répétition d'une année donne le temps à la maturation de faire son ouvre et que l'élève, retardé d'une année, abordera les apprentissages, objets de difficultés ou de blocages, avec des capacités cognitives améliorées. Par ailleurs, comme l'a montré Draelants (2006) en s'entretenant avec des enseignants du secondaire de la Communauté française de Belgique, le redoublement répond à une rationalité pragmatique: il permet notamment de restructurer les groupes d'élèves et, en l'occurrence, d'éviter des effets Robin des Bois consistant à ralentir les progrès des meilleurs pour attendre les élèves en difficulté. Enfin, cette croyance est aussi une représentation sociale: se nourrissant du fait qu'une majorité d'enseignants la partage, au moins à des degrés divers, elle se perpétue, voire se renforce. Ainsi, de jeunes enseignants, informés des résultats de recherche indiquant l'inefficacité de cette pratique, font remarquer que, dans le monde de la pratique, quasiment tout le monde est convaincu de la nécessité de son maintien.

Il s'agit bien d'une croyance "conditionnelle» dans les bienfaits du redoublement. Soucieux de prendre la meilleure décision pour le bien de l'enfant certains enseignants sont assaillis par le doute face à certains cas d'élèves. Ils se deman- 
dent alors, confrontés à certains cas précis, si faire redoubler l'élève équivaut à agir dans son intérêt. À titre d'exemples, on citera deux propos d'enseignants.

C'est toujours délicat. Mais je pense qu'il est nécessaire dans certains cas de faire redoubler un élève, si ça peut contribuer à un meilleur avenir pour lui. [Entretien 18]

Est-ce qu'il va profiter de refaire l'année ou pas? [Entretien 21]

Et c'est bien ce souci du bien de l'élève qui rend la décision difficile.

En tous les cas, c'est toujours une décision difficile à prendre, que ce soit dans un sens ou dans l'autre. [Entretien 55]

Cette croyance conditionnelle dans les bienfaits du redoublement fonctionne comme système de référence en ce sens qu'il opère en tant que schème d'assimilation de la réalité comme l'entendaient Noizet et Caverni (1976): c'est en fonction de cette croyance dans les bienfaits potentiels du redoublement que les enseignants perçoivent l'évolution des redoublants et qu'ils orientent leurs questionnements et, plus globalement, leur réflexion concernant les élèves en grande difficulté. Il n'est pas question pour eux de s'interroger fondamentalement sur la pertinence de cette pratique. Leurs interrogations portent sur l'opportunité de la mettre en œuvre. Ce qui se serait passé si l'élève en grande difficulté avait été promu, n'est quasiment jamais soulevé. Quant aux contre-exemples, les redoublements qui ne produisent pas les effets escomptés, ils sont soit refoulés, soit expliqués par des causes externes (l'élève n'a pas consenti les efforts attendus; il n'a pas reçu le soutien de ses parents). On pourrait penser que la croyance conditionnelle qu'ont les enseignants dans les effets potentiellement positifs du redoublement est munie d'une ceinture d'arguments protecteurs qui, saturés en attributions externes, la rend en quelque sorte infalsifiable. Si nécessaire, des enseignants argueront que, de toute façon, revoir une seconde fois la matière ne peut faire de tort.

Cette croyance dans les bienfaits potentiels du redoublement oriente le questionnement des enseignants. Animés de cette croyance, les enseignants sont logiquement amenés à un double questionnement: quels sont les élèves pour qui le traitement est indiqué et à qui est-il juste de l'appliquer? Le premier questionnement renvoie à une perception des élèves qui constitue en quelque sorte la facette psychologique du jugement qu'opèrent les enseignants, tandis que le second en constitue la facette éthique et débouche sur des dilemmes inéluctablement situés dans les contraintes institutionnelles de l'exercice de la profession enseignante.

Pour assumer le premier questionnement, les enseignants sont conduits à opérer des jugements sur l'état psychologique des élèves en difficulté. Comme on l'a vu, celui-ci prévaut sur les évaluations strictement pédagogiques et, partant, sur les notes scolaires. Celles-ci, par essence malléables, fonctionnent à la fois comme des éléments de validation du jugement psychologique de l'enseignant et outils stratégiques et/ou communicationnels à l'égard des élèves et des parents. 
Qu'ils parlent des élèves ou de leurs familles, les enseignants énoncent des jugements normatifs. D'une part, l'élève est regardé au travers d'un prisme qui renvoie au métier d'élève tel qu'il est idéalisé par l'institution scolaire: enthousiaste, actif, participatif, autonome, soigneux, fiable, motivé, etc. D'autre part, les environnements familiaux sont appréciés en fonction d'une représentation normative de la famille idéale (mais, parfois, qualifiée de «normale»): parents non divorcés, parlant la langue d'enseignement à la maison, ambitionnant pour l'enfant un avenir scolaire jalonné de réussites et, partant, propices à apporter soutien et accompagnement éducatif. Cette double dimension de la perception des élèves peut déboucher sur des raisonnements de compensation ou, à l'opposé, de cumulation. Des enseignants se prononceront pour la réussite d'un élève accumulant les défaillances, mais dont la famille fait preuve d'un potentiel de ressources éducatives importantes. À l'inverse, ils décideront du redoublement d'un élève moyennement faible, mais dont ils estiment que leur famille ne pourra supporter leurs efforts dans le futur.

Le second questionnement s'exprime sous la forme de dilemmes moraux. Comme indiqué ci-dessus, ceux-ci émergent lorsque mérite et talent ne convergent pas. Il est évidemment relativement simple de décider un redoublement lorsque l'élève présente d'importantes lacunes et qu'il ne fait pas de gros efforts pour réussir. Le doute s'immisce dans l'esprit des enseignants lorsqu'ils sont confrontés à des cas d'élèves en difficulté mais méritants, ou avec ceux d'élèves dont les performances sont limites, mais qui exercent leur métier d'élève avec une sorte de désinvolture. Il y a aussi dilemme lorsque l'enseignant considère qu'en décidant le redoublement d'un élève $\mathrm{X}$, il peut aboutir à une reconfiguration du groupe d'élèves qui contribue à une dynamique de classe mieux adaptée au rythme de progrès de la majorité, mais qu'en revanche, cette décision pourrait ne pas être favorable à l'élève concerné. L'enseignant se trouve alors au cœur d'un conflit d'intérêt qui l'oblige à choisir entre l'intérêt du groupe et celui d'un ou deux individus. Ces incertitudes, exprimées à quelques occasions par des enseignants, confirment que, pour certains enseignants au moins, la croyance dans les bienfaits du redoublement n'est pas absolue.

Faut-il parler de professionnalité en ce qui concerne les jugements mobilisés par les enseignants pour décider du redoublement de certains élèves? Cette question interroge les qualités que devraient posséder un jugement professionnel. On répondra affirmativement à la question si l'on considère qu'un jugement est professionnel dès lors que l'enseignant est capable de justifier la décision prise, qu'il a tenu compte de la diversité des facteurs impliqués, qu'il a pris en considération les conditions toujours particulières qui s'appliquent au cas de l'élève en délibération. La réponse sera plus nuancée si l'on adopte la définition de Lafortune (2006). Pour cette auteure, un jugement professionnel suppose un processus cognitif qui «mobilisant différentes considérations issues de son expertise (expérience et formation) aboutit à une décision qui, appuyée sur une collecte d'informations à l'aide de différents moyens, peut être justifiée rationnelle- 
ment». La même auteure souligne qu’ "un jugement professionnel exige rigueur, cohérence et transparence». Or, les entretiens auxquels nous avons procédé révèlent qu'il arrive régulièrement aux enseignants de procéder à ce que Merle (1996) appelle des «arrangements évaluatifs». Ceux-ci se tissent notamment autour de la note, dont nous avons souligné l'usage stratégique qu'en faisaient les enseignants.

La réponse quant à la professionnalité des jugements des enseignants concernant le redoublement et/ou la promotion des élèves, est plus incertaine encore lorsqu'on adopte le point de vue de Legendre (2001). Pour cette chercheuse:

une personne est considérée comme professionnelle lorsqu'elle dispose d'un répertoire de connaissances et d'expériences lui permettant d'évaluer de façon appropriée les situations variées auxquelles l'expose sa pratique, de prendre des décisions éclairées et de les assumer ... quitte à devoir faire des ajustements en cours de route pour tenir compte de l'apport de nouvelles données (p. 18).

Ainsi, selon cette définition, le professionnel dispose de connaissances (et d'expériences) fondant ses jugements et décisions. Or, selon notre analyse, les jugements des enseignants s'appuient essentiellement sur une croyance (ou un réseau de croyances). Quasiment jamais dans leurs explications, ceux-ci n'évoquent leur connaissance des recherches sur le redoublement. Peut-on dès lors considérer qu'ils mobilisent des jugements professionnels alors que leurs processus de réflexion tournent le dos à un des savoirs les mieux établis en sciences de l'éducation: l'inefficacité du redoublement? Certes, on peut s'accorder avec la prémisse de leur raisonnement lorsqu'ils soulignent l'importance des connaissances antérieures dans les apprentissages scolaires, mais il est difficile de s'accorder avec eux lorsqu'ils en inferent l'utilité du redoublement en cas de lacunes importantes à ce niveau. Rappelons également qu'ailleurs, dans les pays nordiques essentiellement, cette croyance (même conditionnelle) n'est pas partagée par les enseignants.

Faut-il alors conclure que les enseignants interrogés dans le cadre de cette recherche manquent de professionnalité? Ce serait exagéré! Il convient, en effet, de rappeler leur souci de prendre en considération la situation toujours singulière de chaque élève dans ses dimensions cognitives, affectives et relationnelles. Leurs décisions de redoublement se présentent dès lors à chaque fois comme un jugement individualisé, qui se veut adapté au cas et à la situation particulière de l'élève. Soucieux de l'intérêt de chaque élève, ils sont fréquemment assaillis de dilemmes qui les obligent à choisir entre le bien particulier de l'élève en difficulté et celui des autres qu'ils craignent de freiner dans leur dynamique développementale.

\section{Notes}

1 Nous adoptons la définition de Richardson (1996) selon lequel les croyances sont des pensées, des prémisses, des propositions ou encore des assertions portant sur des objets et des phénomènes du monde matériel ou mental ; ces pensées ou idées sont supposées possédées par le sujet qui les mobilise ou les formule. Ce qui distingue fondamentalement la 
croyance de la connaissance, c'est la faiblesse des justifications (preuves et/ou arguments) de la validité de la (ou des) proposition(s) retenue(s) ou, au moins, sa (ou leur) plus grande plausibilité par rapport à d'autres conceptions relatives au même objet ou au même phénomène.

\section{Références bibliographiques}

Aubret, J. (1995). Psychologie de l'évaluation. In D. Gaonac'h \& C. Golder (Éd.), Profession enseignant: manuel de psychologie pour l'enseignement (pp. 540-555). Paris: Hachette.

Bless, G., Bonvin, P. \& Schüpbach, M. (2005). Le redoublement scolaire: ses déterminants, son efficacité, ses conséquences. Berne: Haupt.

Bressoux, P. \& Pansu, P. (2003). Quand les enseignants jugent leurs élèves. Paris: Presses Universitaires de France.

Crahay, M. (2007). Peut-on lutter contre l'échec scolaire? (3e éd.). Bruxelles: De Boeck.

Crossley, M. \& Vulliamy, G. (1997). Qualitative educational research in developing countries: current perspectives. New York-London: Garland.

Draelants, H. (2006). Le redoublement est moins un problème qu'une solution: comprendre l'attachement social au redoublement en Belgique francophone. Les Cahiers de Recherche en Education et Formation, 52, 3-21.

Gilly, M. (1980). Maître-élève: rôles institutionnels et représentations. Paris: Presses Universitaires de France.

Grisay, A. (1988). Du mythe de la "bonne école» à la réalité (fuyante) de l' "école efficace». Liège: Service de Pédagogie expérimentale de l'Université.

Hutmacher, W. (1993). Quand la réalité résiste à la lutte contre l'échec scolaire: analyse du redoublement dans l'enseignement primaire genevois. Genève: Service de la recherche sociologique, 36.

Lafortune, L. (2006). Exercice et développement du jugement professionnel, accompagner l'évaluation des apprentissages dans l'école québécoise. Aide à l'apprentissage et reconnaissance des compétences, Fascicule 3, [Document inédit, MELS-UQTR], Consulté le 15 juillet 2007 dans www.uqtr.ca/accompagnement-recherche, sous la rubrique «Accompagner l'évaluation des apprentissages dans l'école québécoise».

Laveault, D. (2005). Le jugement professionnel de l'enseignant: quel en est l'impact sur l'acte d'évaluer? Mesure et Évaluation en Éducation, 28 (2), 93-114.

Legendre, M.-F. (2001). Favoriser l'émergence de changements en matière d'évaluation. Vie Pédagogique, 20, 15-19.

Noizet, G. \& Caverni, J. P. (1976). Psychologie de l'évaluation scolaire. Paris: Presses Universitaires de France.

Merle, P. (1996). L'évaluation des élèves. Enquête sur le jugement professoral. Paris: Presses Universitaires de France.

Perrenoud, P. (1994). Métier d'élève et sens du travail scolaire. Paris: ESF.

Pini, G. (1991). Effets et méfaits du discours pédagogique: échec scolaire et redoublements vus par les enseignants. Éducation et Recherche, 3, 255-272.

Richardson, V. (1996). The role of attitudes and beliefs in learning to teach. In J. Sikula (Éd.), The handbook of research on teacher education (2e éd., pp. 102-119). New York: Macmillan.

Smith, M. L. (1990). Teachers' beliefs about retention. In L. A. Shepard \& M. L. Smith (Éd.), Flunking grades: Research and policies on retention (pp. 16-33). Bristol: Falmer Press.

Tanner, C. K. \& Combs, F. E. (1993). Student retention policy: The gap between research and practice. Journal of Research in Childhood Education, 8 (1), 69-77.

Tomchin, E. M. \& Impara, J. C. (1992). Unraveling teachers' beliefs about grade retention. American Educational Research Journal, 29 (1), 199-223. 
Mots clés: Redoublement, formation d'un jugement complexe, construit représentationnel, conceptions pédagogiques, dilemmes moraux et éthiques, croyances conditionnelles

\section{Warum lassen sie immer wieder wiederholen? Ein Versuch, das Lehrerurteil zu einer Klassenwiederholung zu verstehen}

\section{Zusammenfassung}

Das Entscheiden über eine Klassenwiederholung eines Schülers ist komplex. Es scheint, dass eine Gesamtheit von Elementen den Lehrer zu dieser Massnahme als die am Angemessenste veranlasst. Dieser Artikel versucht, diese Komplexität zu erfassen und zu analysieren. Vorgeschlagen wird, noch präziser den Prozess der Urteilsbildung zu analysieren, der zu dieser Entscheidung führt. Welche Elemente stützen das Urteil, das zu einer Entscheidung für die Wiederholung führt, und welches Gewicht nehmen die verschiedenen Bestandteile in der abschließenden Entscheidung ein? Die vorgestellten empirischen Daten gehen aus Gesprächen mit 41 Lehrern der 6. Grundschulklassen im Kanton Genf im Laufe des Schuljahres 2007-2008 hervor. Ausgehend von den effektiven Entscheidungen einer Lehrperson, sowohl für die Wiederholung eines Schuljahres als auch der Promotionsentscheidung, versuchen diese Gespräche, die Entscheidungsgrundlage des Urteils zu eruieren. Es zeigt sich, dass die persönlichen Überzeugungen, dass diese Klassenwiederholung dem Kind «gut tun wird» zum dominanten Entscheidungskriterium werden. Die Frage, ob dieses Urteil als "professionell» qualifiziert werden kann, wird in der abschliessenden Diskussion behandelt.

Schlagworte: Klassenrepetition, Bildung eines komplexen Urteils, repräsentationelles Konstrukt, pädagogische Konzeptionen, ethische und moralische Dilemmata, bedingte Überzeugungen

\section{Ma perché continuano a bocciare ? Tentativo di comprensione del giudizio degli insegnanti a proposito della bocciatura}

\section{Riassunto}

Decidere di bocciare un allievo è una decisione complessa essendo un insieme di che conducono un insegnante a considerare questa misura. È questa complessità che l'articolo cerca di cogliere e di analizzare. Più precisamente, si propone di analizzare in maniera approfondita l'azione (processo e formazione) che conduce a questa decisione. Quali elementi costitutivi fondano il giudizio alla base della decisione di bocciatura e quale peso prendono questi differenti elementi nella decisione finale? I dati empirici proposti sono il risultato di interviste condotte 
presso 41 insegnanti della 6a elementare nel Canton Ginevra nel corso dell'anno scolastico 2007-2008. Partendo dalle decisioni prese da un insegnante per la bocciatura o la promozione, le interviste tentano di ricostruire la trama decisionale del giudizio in situ. La questione della qualificazione di questo giudizio come "professionale» sarà poi trattata nella discussione finale.

Parole chiave: Bocciatura, formazione di un giudizio complesso, costrutto rappresentazionale, concezione pedagogiche, dilemmi morali e etici, credenze condizionali

\title{
Why do they persevere with grade retaining? An attempt to understand teachers' judgment concerning grade retaining.
}

\begin{abstract}
Grade retention decisions are complex. It seems that a whole set of elements leads teachers to consider this measure as the advisable one to take. This article tries to understand and analyze this complexity. What is proposed is to analyze more thoroughly the action (process and formation) that leads to this judgment. What are the constituents that underlie the judgment in a decision of grade retention and which relevance do these various constituents have in the final decision? The empirical data was collected in interviews with 41 6th grade teachers (primary) in Geneva during the school year 2007-2008. Each teacher is questioned about a promotion and a grade retention decided the previous year. The interviews try to re-tie the decision-making framework of this in situ judgment. The question of qualification of this judgment as "professional» is then tackled in the final discussion.
\end{abstract}

Key words: Grade retention, complex judgment formation, representational construction, educational conceptions, moral and ethics dilemma, conditional faiths. 